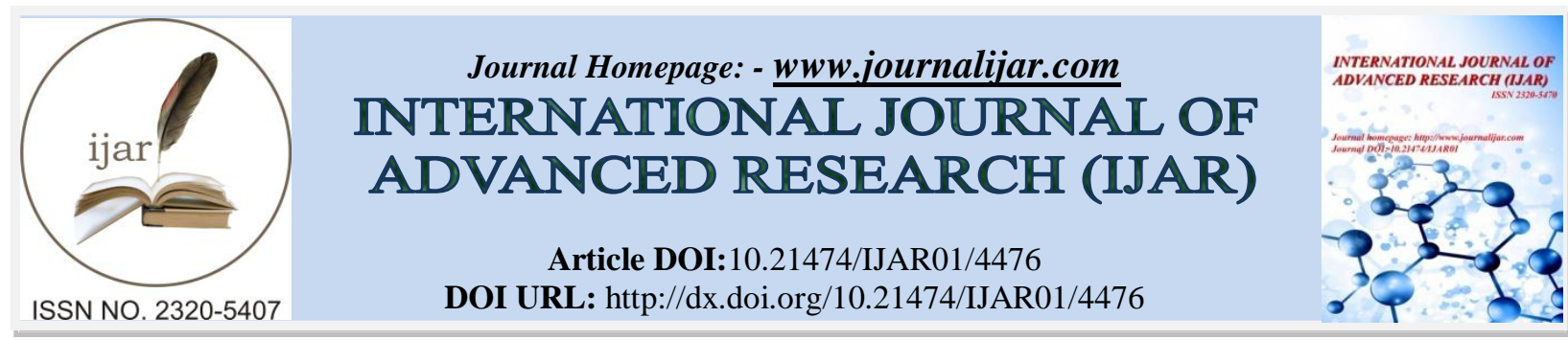

RESEARCH ARTICLE

\title{
CONTROL OF ALEG LAKE WATER QUALITY FOR AGRICULTURAL AND PASTORAL USE (ALEG,
} MAURITANIA).

\section{Yahya Maham Ould Sidi ${ }^{1,2}$, Elhabib El azzouzi ${ }^{1}$, Abdellahi Labidi ${ }^{1}$, Rachid Benaakam ${ }^{1}$, Abdlekrim Bellouchou $^{1}$, Yahya Mkadmi ${ }^{1}$, M.S. kankou ${ }^{2}$, Brahim Ahmed Dick ${ }^{2,3}$, Mohamed avelwatt ${ }^{2}$ and ${ }^{*}$ Mohamed Fekhaoui ${ }^{1}$.}

1. Laboratoire de Pollution, ecotoxicologie et risques sanitaires, Faculté des Sciences, Université Mohammed V de Rabat. Avenue Iben Batota . B, P: 703 : 10106, Rabat-Agdal-Maroc.

2. Laboratoire de l'unité eau, Pollution et environnement .FST, Université de Nouakchott.

3. Laboratoire de chimie des eaux institut National des recherches en sante public (INRSP) en Mauritanie.

\section{Manuscript Info}

Manuscript History

Received: 13 April 2017

Final Accepted: 15 May 2017

Published: June 2017

Key words:-

Qualite, physico-chimie, Eau, Lac d'Aleg, Mauritanie.

\section{Abstract}

Dans ce travail, nous étudions l'hydrochimie des eaux de surface du lac d'Aleg en Mauritanie. Après plusieurs années d'exploitation domestique, industrie et agricole, outre les changements climatiques, Il est intéressant de s'assurer de la qualité de l'eau. avec cette intention, nous avons essayé de réaliser une analyse physico-chimique. évaluation, pour avoir une idée des probabilités de pollution et de l'effet de la variation saisonnière du climat sur l'eau de cette rivière.

Pour cela un échantillonnage a été effectué à saison sèche de 2017 aux 10 stations sur les deux rives du lac. Les paramètres physicochimiques suivants sont: $\mathrm{T}^{\circ} \mathrm{C}, \mathrm{pH}$, Turbidité, C.E, TDS, dureté totale (TH), $\mathrm{Ca}^{2+}, \mathrm{Mg}^{2+}, \mathrm{Na}^{+}, \mathrm{K}^{+}, \mathrm{NH}_{4}^{+}, \mathrm{Cl}^{-}, \mathrm{NO}_{2}^{-}, \mathrm{NO}_{3}^{-}, \mathrm{HCO} 3-, \mathrm{CO} 2, \mathrm{SO}^{2-}$ ${ }_{4}$, Oxydabilité, TA, TAC, Fer, $\mathrm{Cu}, \mathrm{Al}, \mathrm{Pb}, \mathrm{Cr}$ et matières en suspension (MES). Ces mesures comprennent l'analyse volumétrique, spectroscopique, spectrophotomètres d'absorption atomique à four. L'exploitation statistique des résultats et leur comparaison avec les normes européennes de potabilité de l'eau a montré que Il ya une véritable détérioration de la qualité de l'eau du lac.

Les résultats de la présente étude ont montré que la concentration de la quasi-totalité des paramètres physico-chimiques répond aux normes en vigueur avec une turbidité variante de 141 NTU et 771 NTU, ce qui en relation avec les MES qui présente une valeur maximal de $505 \mathrm{mg}$ $/ \mathrm{L}$, et les valeurs du MO minimal de $0,19 \mathrm{mg} / \mathrm{L}$. et une valeur maximale de $1.22 \mathrm{mg} / \mathrm{L}$. La teneur en nitrite varie entre $0,05 \mathrm{mg} / 1$ et $1,6 \mathrm{mg} / 1$ avec une moyenne d'environ $0,475 \mathrm{mg} / 1$ qui dépasse la norme $(0,1 \mathrm{mg} / \mathrm{l})$. La tenure en ammonium varie entre $0,00 \mathrm{mg} / 1$ et $0,4 \mathrm{mg} / 1$ avec une moyenne d'environ $0,14 \mathrm{mg} / 1$ qui dépasse la norme $(0,03 \mathrm{mg} / \mathrm{l})$.

Un contrôle de qualité de l'eau du lac doit être nécessaire et mis à jour notamment la réduction des effluents agricole et pastorale de cette rivière. 


\section{Introduction:-}

Les ressources en eau occupent un rôle important dans le développement des différents secteurs dans n'importe quel pays. La surface l'eau est susceptible d'être utilisée comme les eaux potables et l'eau d'irrigation [1]. C'est le cas du lac d'Aleg qui joue un rôle partie importante comme source primaire d'eau de surface pour la Mauritanie. De plus, la lac est la source d'approvisionnement de ville d'Aleg en plusieurs villages dans la zone du Brakna par l'eau potable et l'eau de irrigation. [2].

Dans ce pays où le climat est aride et sec, une gestion des ressources en eau semble nécessaire. En effet, faible la pluviométrie, la nature géologique du pays et l'importance de l'évaporation rendent le pays faible en eau ressources. Le lac d'Aleg, situé dans la région du Brakna, au sud-ouest de la Mauritanie (est caractérisé par un climat chaud (températures moyennes annuelles supérieures à $35^{\circ} \mathrm{C}$ ) de type saharo sahélien avec une saison sèche qui dure 8 mois (de novembre à juin) [3]. L'hivernage ou saison des pluies qui lui succède dure généralement quatre mois, avec un pic de pluviométrie en août (250-400 mm de pluies/an).

La dynamique de remplissage du lac d'Aleg dépend directement pour l'essentiel des écoulements intermittents de l'oued Ketchi, cours d'eau endoréique le plus important de la région du Brakna ; ces écoulements n'ont lieu que pendant la saison des pluies de juillet à octobre. L'eau termine sa course dans le lac, qui est une dépression peu profonde. [4]

Le lac d'Aleg, dont la superficie en eau varie entre 0 et $60 \mathrm{~km}^{2}$ (Sud-ouest de la Mauritanie, région du Brakna). Situé en région aride, le lac est une zone humide fragile. Elle fait actuellement l'objet d'une pression démographique importante due à l'arrivée des nomades victimes de la sécheresse croissante du Sahel. L'étude du fonctionnement hydraulique de cette zone est en cours de réalisation, pour parvenir à une relative stabilité du niveau des eaux du lac [1].

Actuellement et depuis de nombreuses années, cette rivière vitale a exposé des contraintes qui menacent ses qualités physiques, chimiques et métalliques. Avec divers points géographiques, cette voie d'eau reçoit des rejets d'eaux usées domestiques et industrielles [3].

À ce jour, l'état des rivières et des estuaires africains a été peu étudié, alors qu'ils sont le lieu de démographiques et reçoivent un volume croissant de déchets, en particulier des eaux usées domestiques.

L'objectif de cette étude est d'évaluer la qualité de l'eau du lac d'Aleg en Mauritanie par un espace physico-chimique de surveillance et saisonnier.

\section{Étude Environnementale:- \\ Présentation de La Zone D'étude:-}

La République islamique de Mauritanie est 1025520 kilomètres carrés, et sa position est entre 15 et 27 e nord Degré de latitude. Il a des frontières avec l'Algérie $(463 \mathrm{~km})$, le Maroc $(1561 \mathrm{~km})$, le Mali $(2237 \mathrm{~km})$ et le Sénégal. Il est encadré dans À l'ouest par l'océan Atlantique (700 km) (Figure 1).

La Préfecture du Brakna compte 240.000 habitants, soit $10 \%$ de la population nationale établis dans cinq départements administratifs couvrant 3\% du territoire national. Cette population est à plus de $95 \%$ de sédentaires. Si, les départements d'Aleg et de Magta-Iahjar ont des densités les plus faibles (superficies plus étendues), les populations sont presque toutes regroupées autour du lac d'Aleg [3]. Aleg est une ville coloniale qui a été créee au début du XX ${ }^{\mathrm{e}}$ siècle (1903-1904) et qui est devenue chef-lieu de la région du Brakna. Ce rang administratif lui confère un niveau d'équipements élevé pour une ville mauritanienne, d'environ 7000 habitants. L'activité économique de la région est essentiellement tournée vers l'agriculture qui génère un artisanat local (petites échoppes en tous genres, marchés, foire aux caprins et ovins, etc.) [2]. La ville connaît également une activité commerciale favorisée par sa position sur la Route de l'Espoir, route goudronnée qui a permis le désenclavement des régions du sud de la Mauritanie et qui relie la capitale Nouakchott à Néma.

Le lac d'Aleg, situé dans la région du Brakna, au sud-ouest de la Mauritanie (figure 1) est caractérisé par un climat chaud (températures moyennes annuelles supérieures à $35^{\circ} \mathrm{C}$ ) de type saharo sahélien avec une saison 
sèche qui dure 8 mois (de novembre à juin) [3]. L'hivernage ou saison des pluies qui lui succède dure généralement quatre mois, avec un pic de pluviométrie en août. La dynamique de remplissage du lac d'Aleg dépend directement pour l'essentiel des écoulements intermittents de l'oued Ketchi, cours d'eau endoréique le plus important de la région du Brakna; ces écoulements n'ont lieu que pendant la saison des pluies de juillet à octobre. L'eau termine sa course dans le lac, qui est une dépression peu profonde. [4]. Le lac d'Aleg, dont la superficie en eau varie entre 0 et $60 \mathrm{~km}^{2}$ (Sud-ouest de la Mauritanie, région du Brakna). Situé en région aride, le lac est une zone humide fragile. Elle fait actuellement l'objet d'une pression démographique importante due à l'arrivée des nomades victimes de la sécheresse croissante du Sahel. L'étude du fonctionnement hydraulique de cette zone est en cours de réalisation, pour parvenir à une relative stabilité du niveau des eaux du lac [1].

Dans notre étude, nous avons choisi comme site la zone du lac de la rivière, précisément la ville d'aleg Mauritanie pour plusieurs les raisons. C'est la ville la plus dynamique de la zone et le milieu étudié reçoit les rejets domestiques et les déchets industriels.

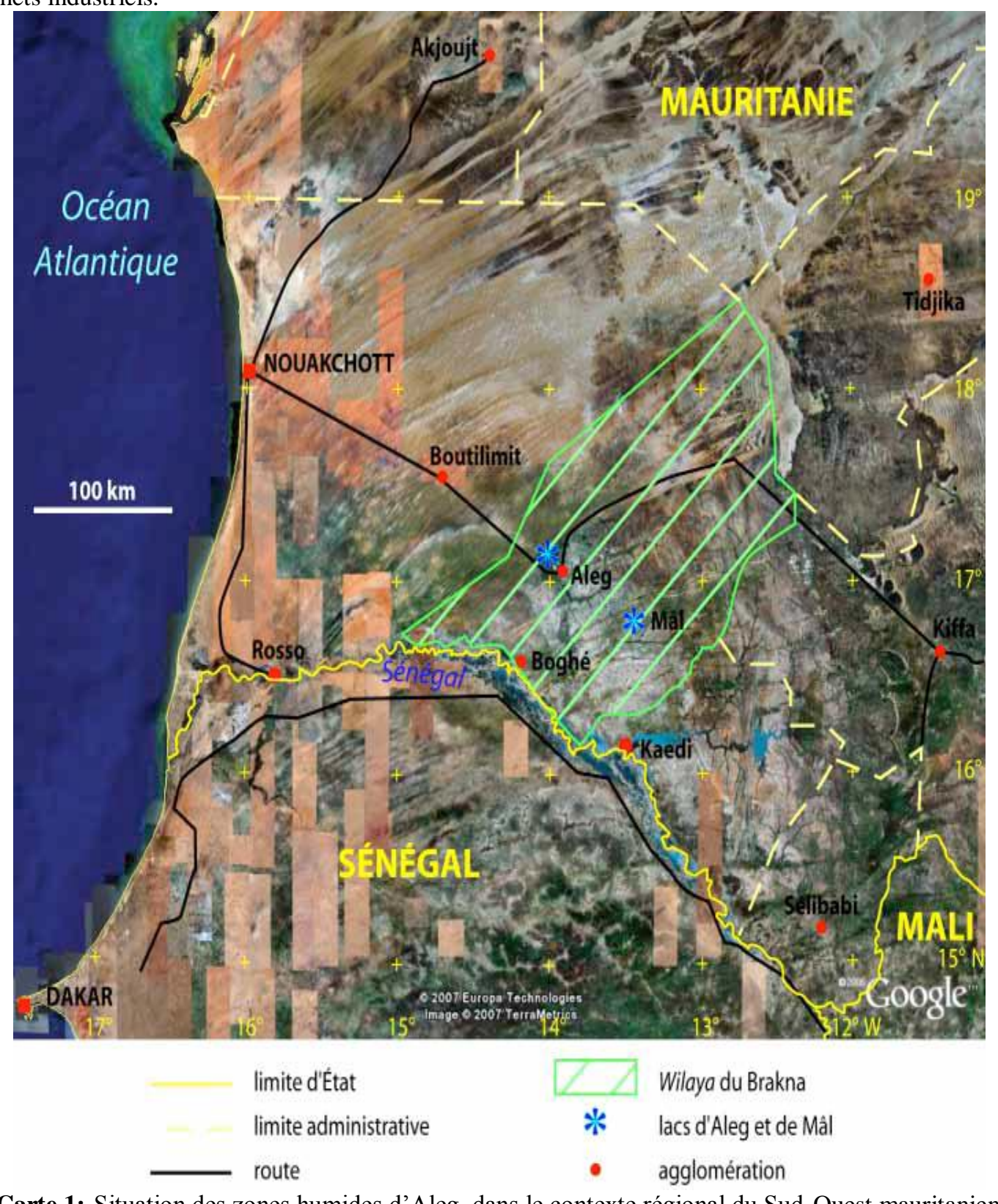

Carte 1:-Situation des zones humides d'Aleg dans le contexte régional du Sud-Ouest mauritanien. 


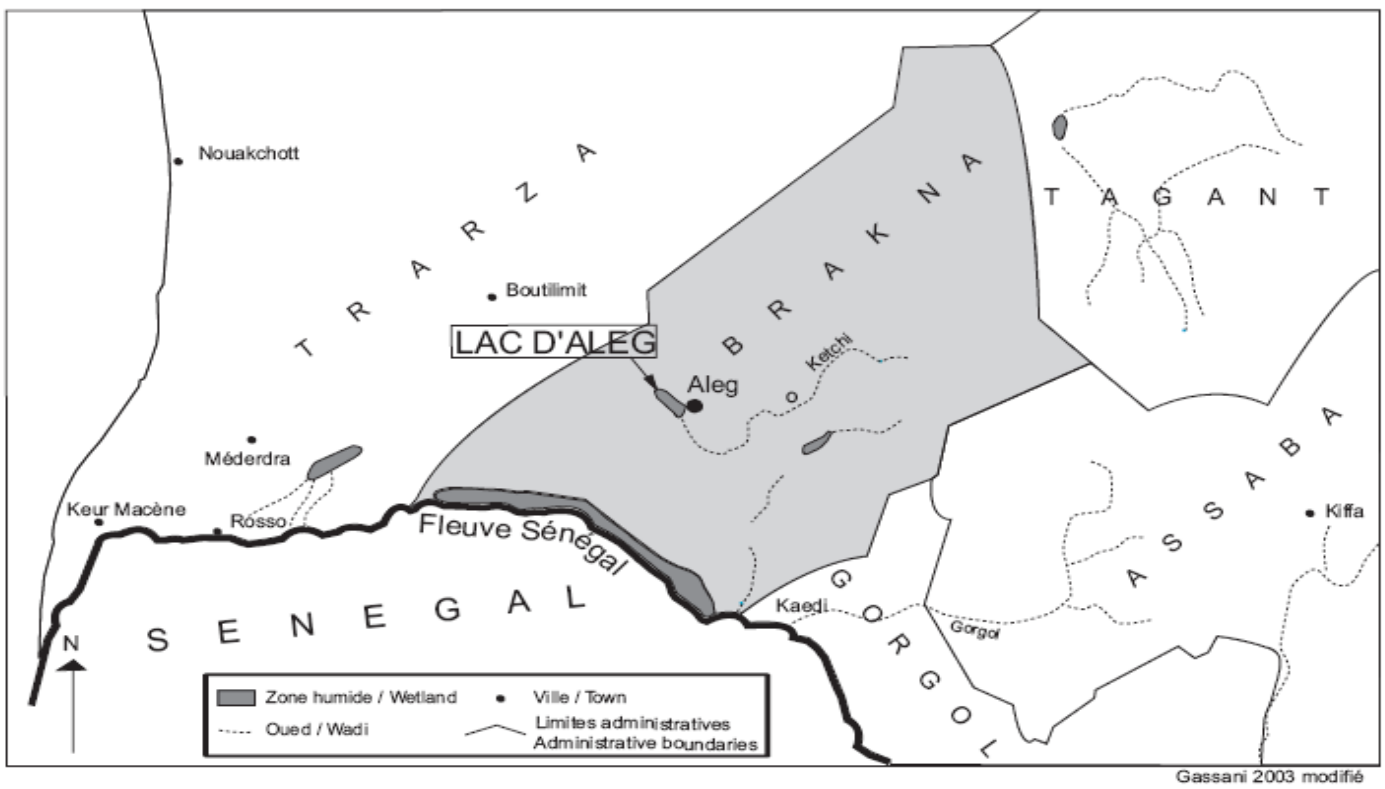

FIGURE 1 Localisation de la zone d'étude. Location map of the study area.

\section{Choix Des Sites D'échantillons:-}

Pour évaluer la qualité de l'eau du lac, on a choisi dix stations d'échantillonnage situées sur les deux rives du lac. Ces stations ont été sélectionnées en fonction de leur accessibilité, de leur proximité et selon les activités agricoles et pastorales. Sources de pollution et leur répartition géographique comme suite:

Tableau 1:-Echantillonnage Eau du Lac d'Aleg en Mauritanie 04 Février 2017

\begin{tabular}{|c|c|c|c|}
\hline & & \multicolumn{2}{|c|}{ Coordonnées GPS } \\
\hline Nom local & Code Eau & $\mathrm{X}(\mathrm{N})$ & $\mathrm{Y}(\mathrm{W} 0)$ \\
\hline Mechraa & Alg 01 & 1708813 & 1398435 \\
\hline Akreraye & Alg 02 & 1709119 & 1398634 \\
\hline Mechraa veived & Alg 03 & 1710479 & 1398811 \\
\hline Dwalek & Alg 04 & 1711913 & 1399449 \\
\hline Mechraa elbel & Alg 05 & 1714439 & 1402297 \\
\hline Engabe & Alg 06 & 1714033 & 1403505 \\
\hline Mechraa Levreiwatt1 & Alg 07 & 1713430 & 1404510 \\
\hline Mechraa Levreiwatt2 & Alg 08 & 1712545 & 1403948 \\
\hline Mechraa Lehjare & Alg 09 & 1711019 & 1401345 \\
\hline Avreiraye & Alg 10 & 1708844 & 1399225 \\
\hline
\end{tabular}

\section{Equipement et Methodes:-}

\section{Échantillonnage:-}

Le prélèvement d'eau du lac d'Aleg a été effectué en Février, dans la saison sèche du partenaire 2017.

Ces prélèvements ont été effectués dans des bouteilles en plastique de 1 litre, Propres, rincés au préalable par l'eau de prise, mis en immersion totale dans l'eau de la rivière et ils sont hermétiquement remplis afin d'éviter toute contamination (Voir photo1). 


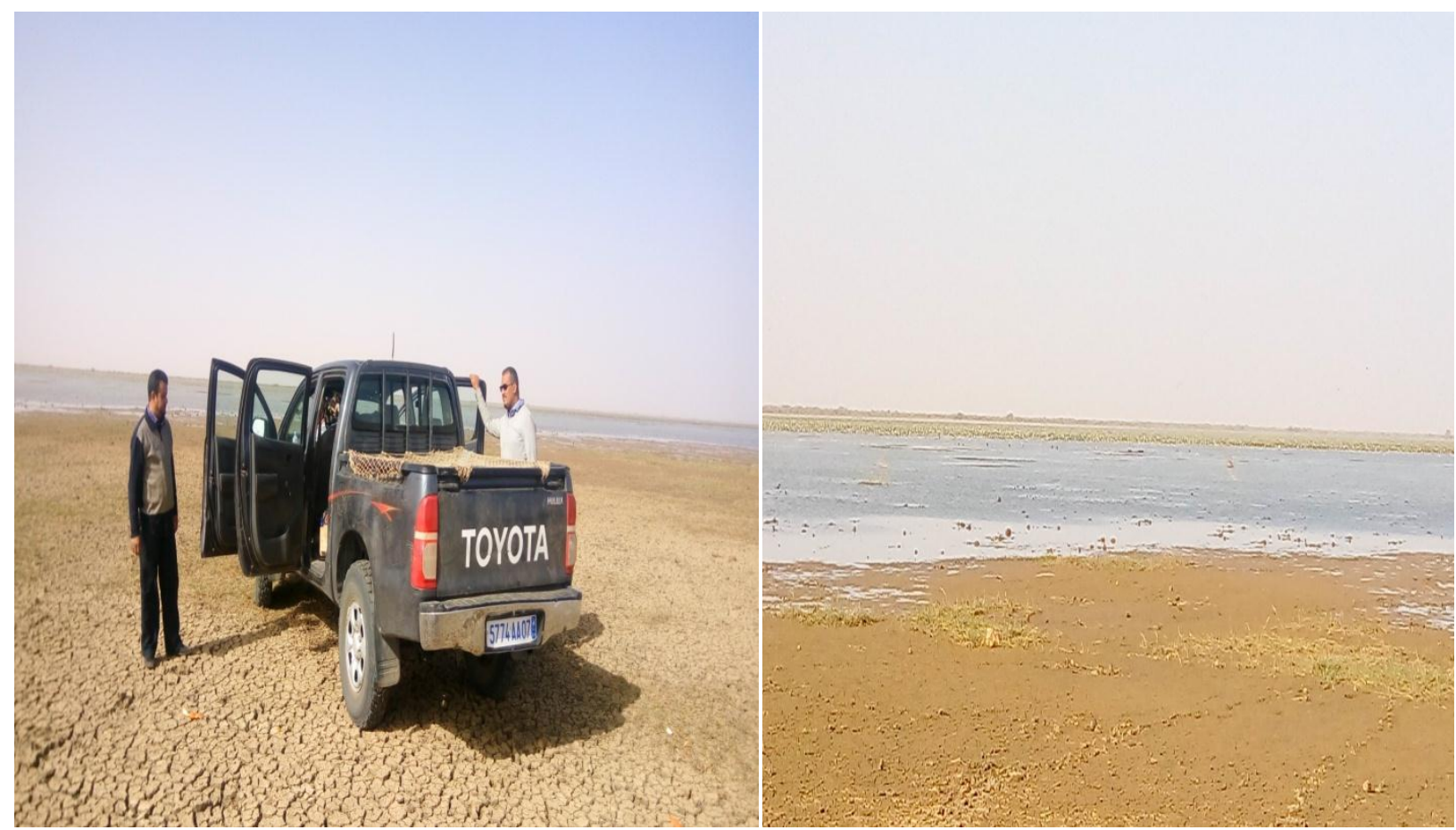

Photo 1 : la mission d'échantillonnage du lac d'Aleg en Mauritanie Février 2017

\section{Méthodes D'analyse:-}

Dans le même temps de l'échantillon d'essai de l'eau, on a procédé à la mesure de la température de l'eau et le pH en utilisant un pH-mètre de type HANNA instrument HI 8314.La conductivité électrique (C.E) a été mesurée, en utilisant un conductimètre du type HANNA instrument HI 8733.La Turbidité a été mesurée, en utilisant un turbidimètre du type HANNA instrument HI 2100.Nitrates (NO3-), les nitrites (NO2), l'ammonium (NH4+) Et des sulfates (SO42-), fer, cuivre, Aluminium, sont dosés par un Spectrophotomètre UV-Visible (WEG 7100).

Les métaux lourds comme le plomb $(\mathrm{Pb})$ et Chrome $(\mathrm{Cr})$ sont dosés par photomètre d'absorption atomique à four marque TOMAS.

Les métaux alcalins sodium $(\mathrm{Na}+)$ et potassium $(\mathrm{K}+)$ sont dosés par photomètre de flamme. Le titre alcalin $(\mathrm{TA})$, complet (TAC), la dureté totale $(\mathrm{HT})$ calcium $(\mathrm{Ca} 2+)$, le magnésium $(\mathrm{Mg} 2+)$, le chlorure $(\mathrm{Cl}-)$, l'oxydabilité, Bicarbonates (HCO3-), sont dosés par la méthode volumétrique Mohr en présence de milieux sélectifs [12]. La détermination de La matière en suspension subit la filtration d'un échantillon d'eau sur un filtre de porosité ( 0 , $45 \mu \mathrm{m})[12]$.

\section{Resultats ét Discussion:-}

Ces résultats sont exploités par l'analyse statistique descriptive en termes d'analyse en composantes principales (ACP), qui Est une technique d'exploration.

La qualité physico-chimique de l'eau brute du lac d'Aleg en Mauritanie du a été suivie par le biais de l'analyse de l'eau récoltée au niveau de dix(10) sites :

Evolution des paramètres physiques

Tableau 2:-Résultats des paramètres physiques de l'eau.

\begin{tabular}{|c|c|c|c|c|}
\hline Paramètre & Unité & Minimum & Maximum & Moyenne \\
\hline Température & ${ }^{\circ} \mathbf{C}$ & $\mathbf{2 0}$ & $\mathbf{2 4}$ & $\mathbf{2 3 , 2 8}$ \\
\hline pH & -- & $\mathbf{7 , 0 2}$ & $\mathbf{7 , 6 2}$ & $\mathbf{7 , 4 2}$ \\
\hline Conductivité & $\boldsymbol{\mu S} / \mathrm{cm}$ & $\mathbf{3 3 9}$ & $\mathbf{7 0 2}$ & $\mathbf{4 5 2}$ \\
\hline TDS & $\mathrm{mg} / \mathbf{l}$ & $\mathbf{1 6 9}$ & $\mathbf{3 5 1}$ & $\mathbf{2 2 6}$ \\
\hline Turbidité & $\mathrm{NTU}$ & $\mathbf{1 4 1}$ & $\mathbf{7 7 1}$ & $\mathbf{4 6 7}$ \\
\hline MES & $\mathrm{mg} / \mathbf{l}$ & $\mathbf{1 5 , 8}$ & $\mathbf{5 0 5}$ & $\mathbf{1 8 5}$ \\
\hline Résidu à sec 102 ${ }^{\circ} \mathrm{C}$ & $\mathrm{mg} / \mathbf{l}$ & $\mathbf{0 , 0 9}$ & $\mathbf{1 , 7 3}$ & $\mathbf{0 , 7 2 7}$ \\
\hline
\end{tabular}




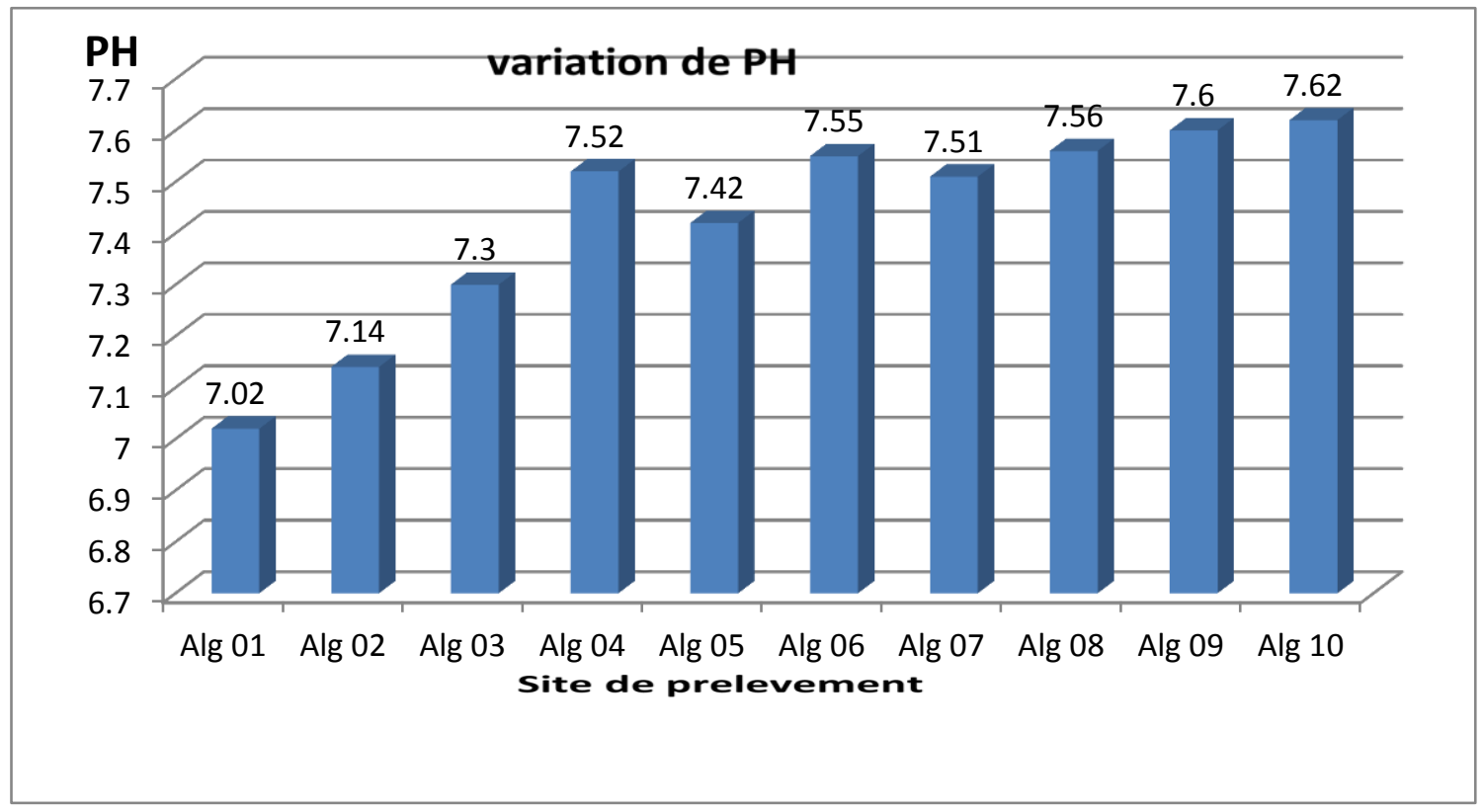

Figure 2 : Variation de $\mathbf{p H}$

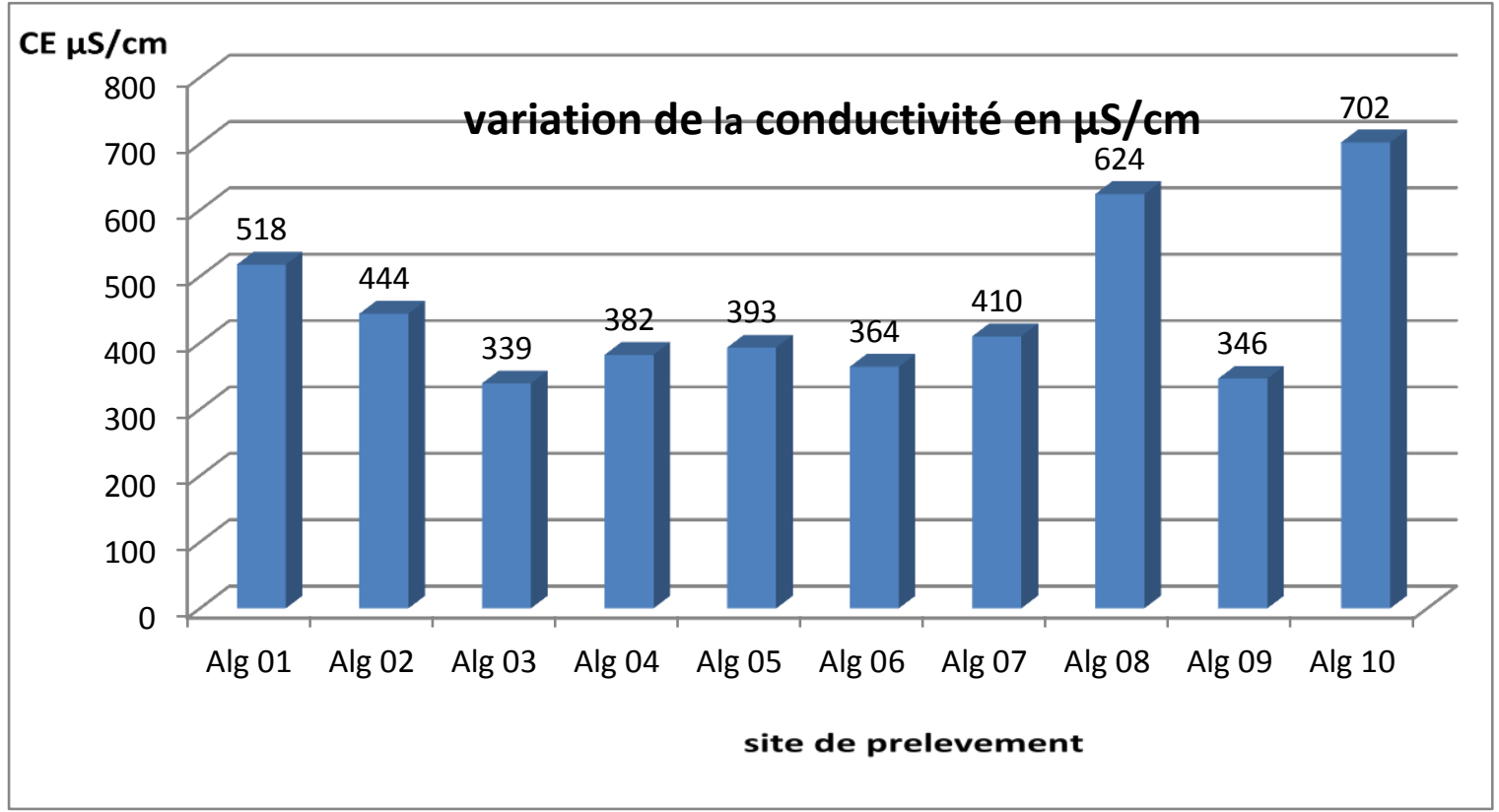

Figure 3 : Variation de la conductivité en $\mu \mathrm{S} / \mathrm{cm}$ 


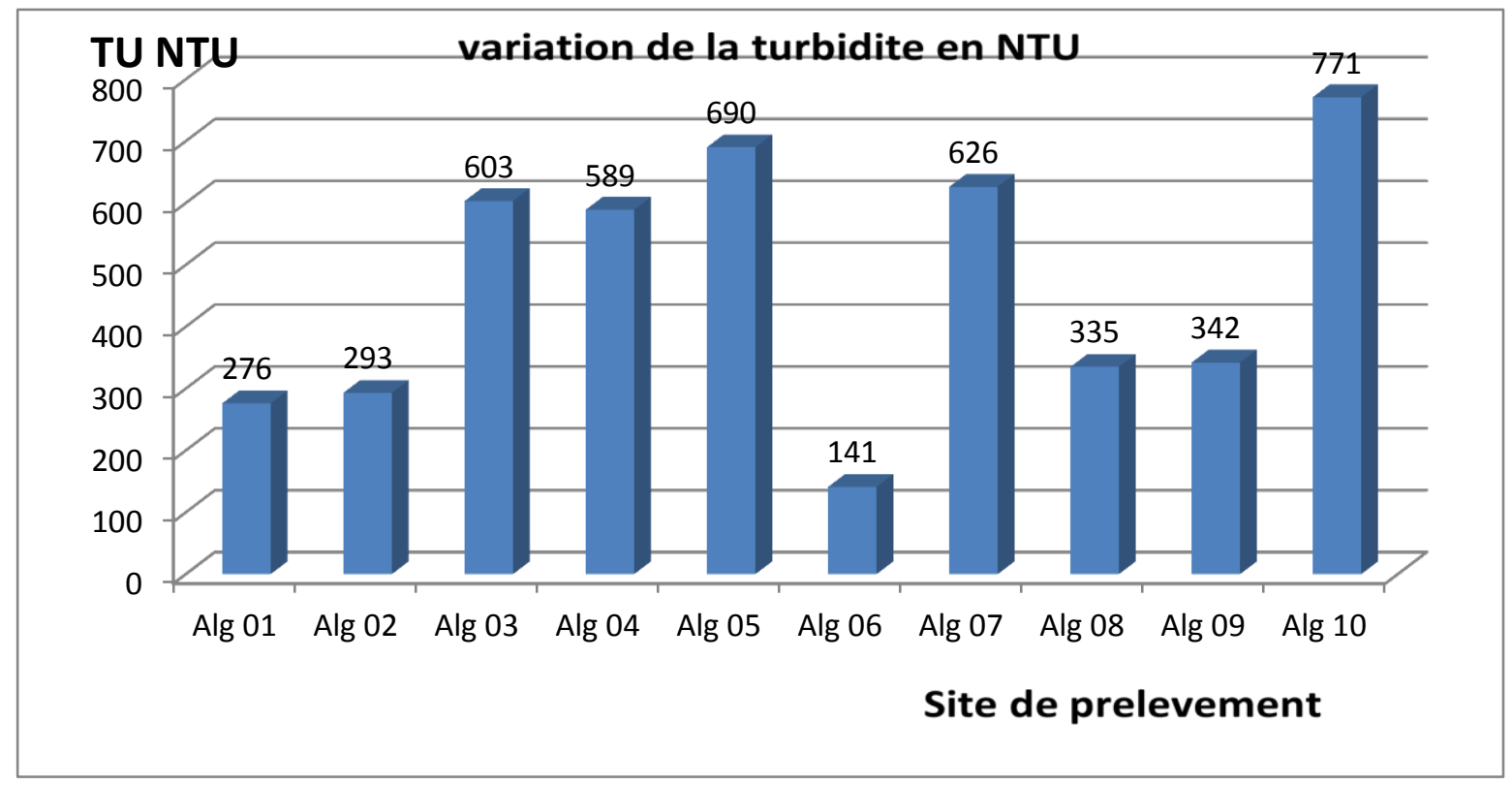

Figure 4 : Variation de la turbidité en NTU

Les analyses effectuées montrent que la température de l'ensemble des échantillons est comprise entre $20^{\circ} \mathrm{C}$ et $24^{\circ} \mathrm{C}$ (Tableau 2). Ainsi que le $\mathrm{pH}$ de l'ensemble des échantillons est compris entre 7,02 et 7,62 (Tableau 2). En ce qui concerne la minéralisation de l'eau brute du lac, les mesures de la conductivité de l'ensemble des échantillons montrent qu'elles sont comprises entre $339 \mu \mathrm{S} / \mathrm{cm}$ et $702 \mu \mathrm{S} / \mathrm{cm}$ (Tableau 2). Les mesures de la TDS de l'ensemble des échantillons montrent qu'elles sont comprises entre $169 \mathrm{mg} / \mathrm{L}$ et $351 \mathrm{mg} / \mathrm{L}$ (Tableau 2).

Les valeurs de la turbidité sont situées entre 141 NTU et 771 NTU (Tableau 2) ainsi que Les valeurs du MES comprise entre $15.8 \mathrm{mg} / \mathrm{l}$ et $505 \mathrm{mg} / 1$ (Tableau 2).

Tableau 3:- Résultats des paramètres chimiques de l'eau

\begin{tabular}{|c|c|c|c|c|}
\hline Paramètres & Unité & Minimum & Maximum & Moyenne \\
\hline Dureté total & ${ }^{\circ} \mathbf{f}$ & $\mathbf{5 . 2 0}$ & $\mathbf{1 2 . 2}$ & 7.50 \\
\hline Calcium & $\mathrm{mg} / \mathrm{l}$ & $\mathbf{1 6 . 0 3}$ & $\mathbf{5 4 . 5 0}$ & 35.75 \\
\hline Magnésium & $\mathrm{mg} / \mathrm{l}$ & $\mathbf{1 9 . 4 4}$ & $\mathbf{4 5 . 2 0}$ & $\mathbf{2 9 . 1 6}$ \\
\hline Sodium & $\mathrm{mg} / \mathrm{l}$ & $\mathbf{2 5}$ & $\mathbf{5 1}$ & 32 \\
\hline Potassium & $\mathrm{mg} / \mathrm{l}$ & $\mathbf{2 0}$ & $\mathbf{3 5}$ & $\mathbf{2 4 . 4}$ \\
\hline Chlorure & $\mathrm{mg} / \mathrm{l}$ & $\mathbf{1 7 . 7 5}$ & $\mathbf{1 0 3}$ & $\mathbf{4 1 . 5 3}$ \\
\hline Titre Alcalin & ${ }^{\circ}$ & $\mathbf{0 . 0 0}$ & $\mathbf{0 . 0 0}$ & $\mathbf{0 . 0 0}$ \\
\hline Titre Alcalin Complet & ${ }^{\circ} \mathbf{f}$ & $\mathbf{1 0 . 5}$ & $\mathbf{2 7 . 5}$ & $\mathbf{1 8 . 7 7}$ \\
\hline Oxydabilité & $\mathrm{mgd}^{\prime} \mathbf{O} / \mathbf{l}$ & $\mathbf{0 . 1 9}$ & $\mathbf{1 . 2 2}$ & $\mathbf{0 . 5 2}$ \\
\hline L'anhydre carbonique & $\mathbf{m g} / \mathbf{l}$ & $\mathbf{7 9 . 2}$ & $\mathbf{3 5 . 2}$ & $\mathbf{5 6 . 7 6}$ \\
\hline
\end{tabular}

Les teneurs en Sodium et en potassium des échantillons varient respectivement de $25 \mathrm{mg} / \mathrm{L}$ à $51 \mathrm{mg} / \mathrm{L}$ et de $20 \mathrm{mg} / \mathrm{L}$ à $35 \mathrm{mg} / \mathrm{L}$ (Tableau 3). Les teneurs en TH, Calcium et Magnésium varient entre 5.20 et 12.02, $16.03 \mathrm{mg} / \mathrm{L}$ et 54.50 $\mathrm{mg} / \mathrm{L}$ et de $19.44 \mathrm{mg} / \mathrm{L}$ et $45.20 \mathrm{mg} / \mathrm{L}$ (Tableau 3). Les teneurs en Chlorure et Carbonates varient entre $17.75 \mathrm{mg} / \mathrm{L}$ et $103 \mathrm{mg} / \mathrm{L}$ et $10.5^{\circ} \mathrm{f}$ et $27.5^{\circ} \mathrm{F}$ (Tableau 3).Les teneurs de l'oxydabilité en mgd'O2/1 varient entre 0.19 et 1.22 (Tableau 3). Les teneurs de l'anhydre carbonique en $\mathrm{mg} / \mathrm{l}$ varient entre 79.2 et 35.2 (Tableau 3). 


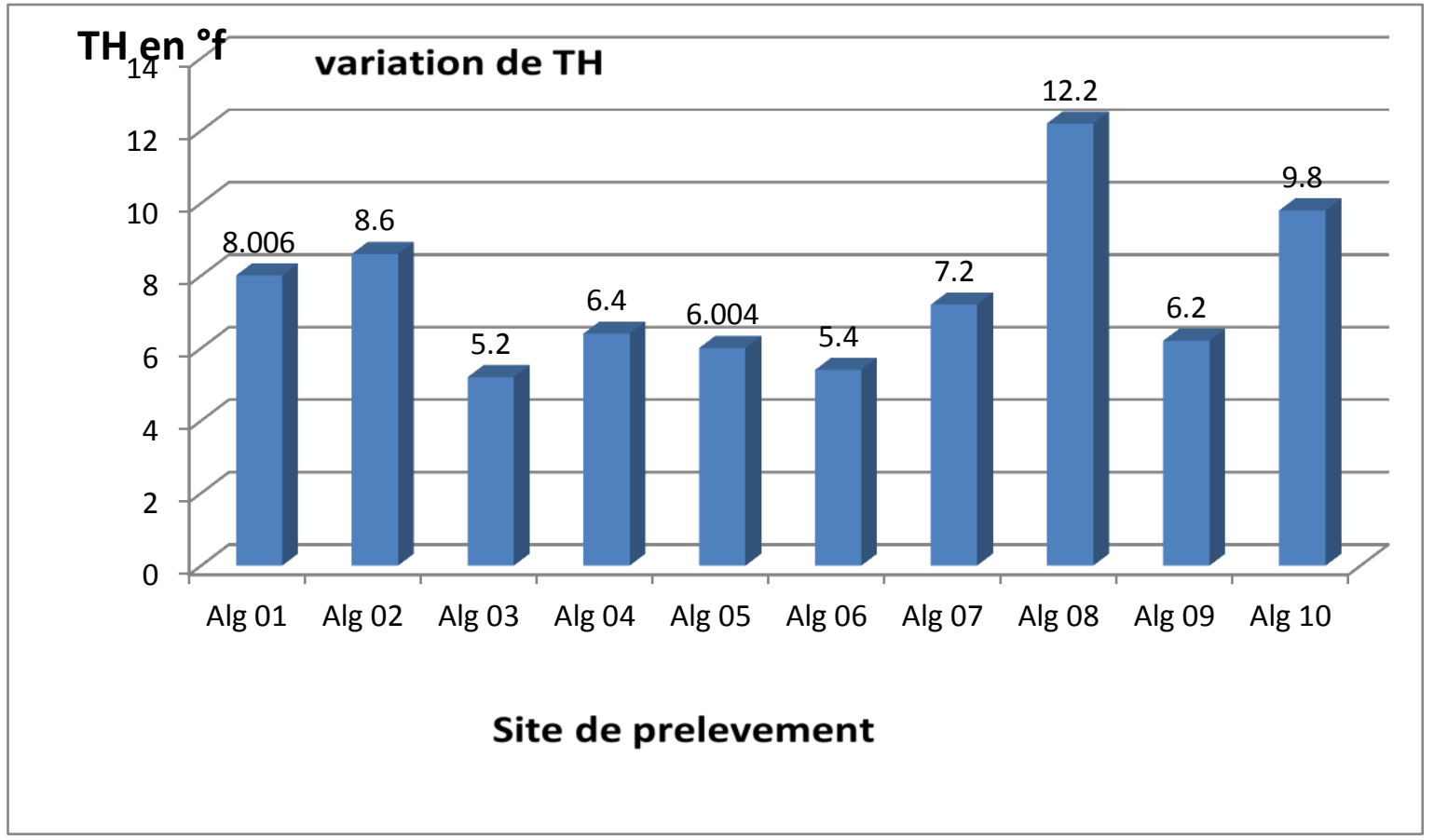

Figure5 : Variation du TH en ${ }^{\circ} f$

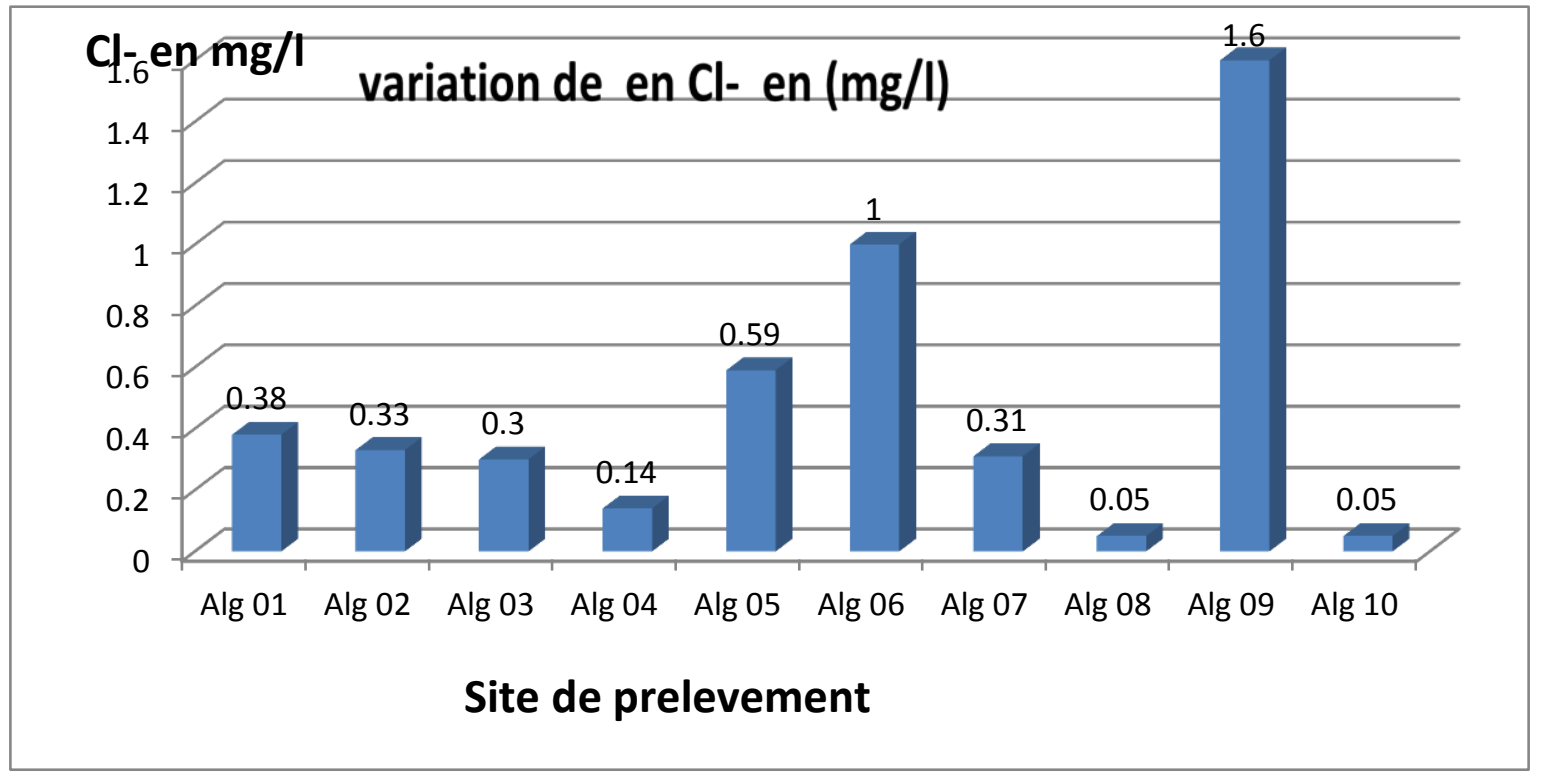

Figure 6 : Variation du chlorure en mg/l 
Tableau4:-Résultats des paramètres toxiques de l'eau

\begin{tabular}{|c|c|c|c|c|}
\hline Paramètres & Unité & Minimum & Maximum & Moyenne \\
\hline Nitrates & $\mathrm{mg} / \mathrm{l}$ & $\mathbf{3 . 8}$ & $\mathbf{1 5}$ & $\mathbf{9 . 5 4}$ \\
\hline Nitrites & $\mathrm{mg} / \mathrm{l}$ & $\mathbf{0 . 0 5}$ & $\mathbf{1 . 6 0}$ & $\mathbf{0 . 4 7}$ \\
\hline Ammonium & $\mathrm{mg} / \mathrm{l}$ & $\mathbf{0 . 0 0}$ & $\mathbf{0 . 4}$ & $\mathbf{0 . 1 4}$ \\
\hline Sulfates & $\mathrm{mg} / \mathrm{l}$ & $\mathbf{9 1}$ & $\mathbf{2 8 . 3}$ \\
\hline
\end{tabular}

En ce qui concerne les composés azotés (nitrates, nitrites et ammonium), leurs teneurs sont varies : Les teneurs en Nitrate et en Nitrites des échantillons varient respectivement de 3.5mg/L à $15 \mathrm{mg} / \mathrm{L}$ et de $0.05 \mathrm{mg} / \mathrm{L}$ à $1.6 \mathrm{mg} / \mathrm{L}$ (Tableau 4). Les teneurs en Ammonium des échantillons varient 0.00mg/L à $0.4 \mathrm{mg} / \mathrm{L}$ (Tableau 4).

Les teneurs en Sulfates des échantillons varient de 9 mg/L à $61 \mathrm{mg} / \mathrm{L}$ (Tableau 4).

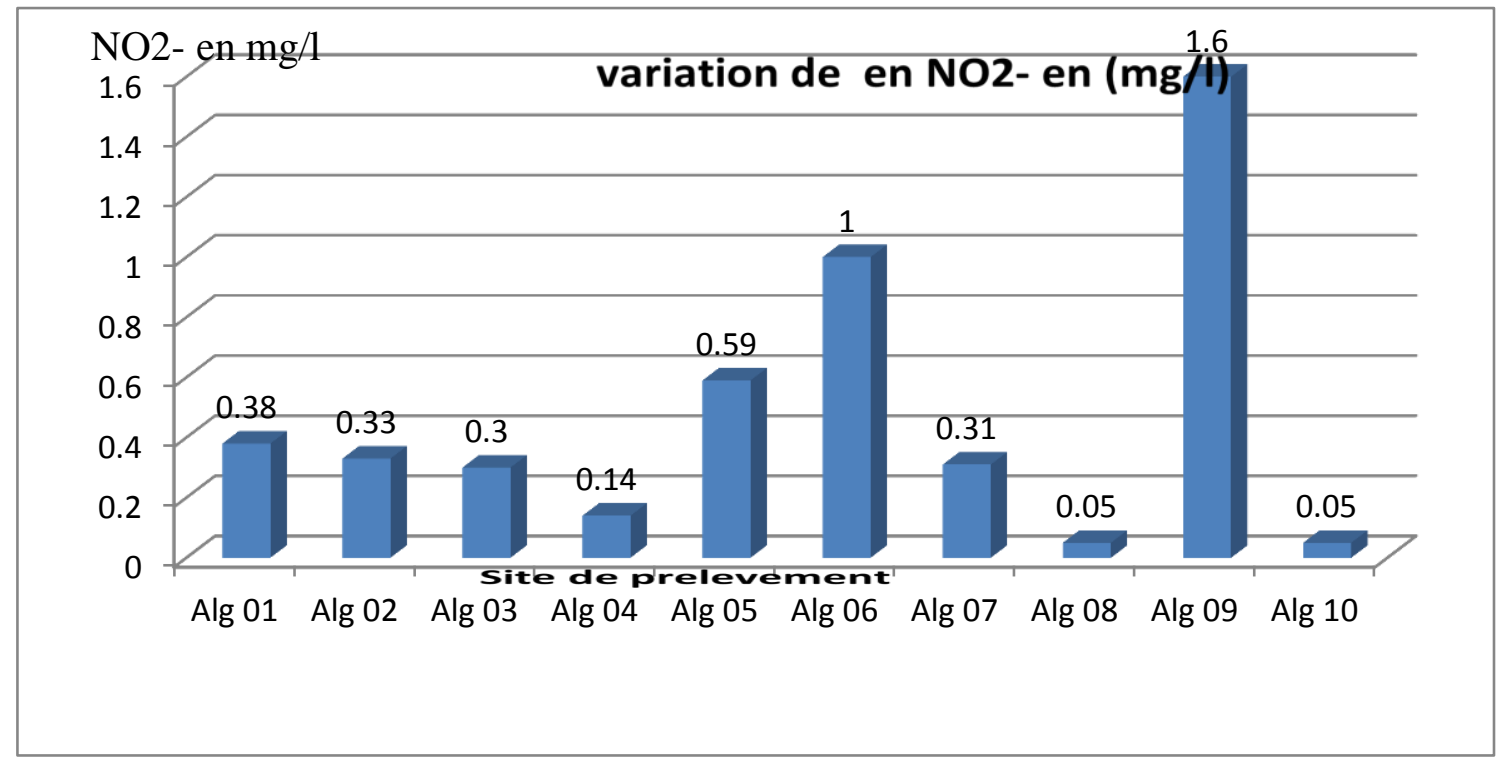

Figure 7 de Nitrite en mg/l

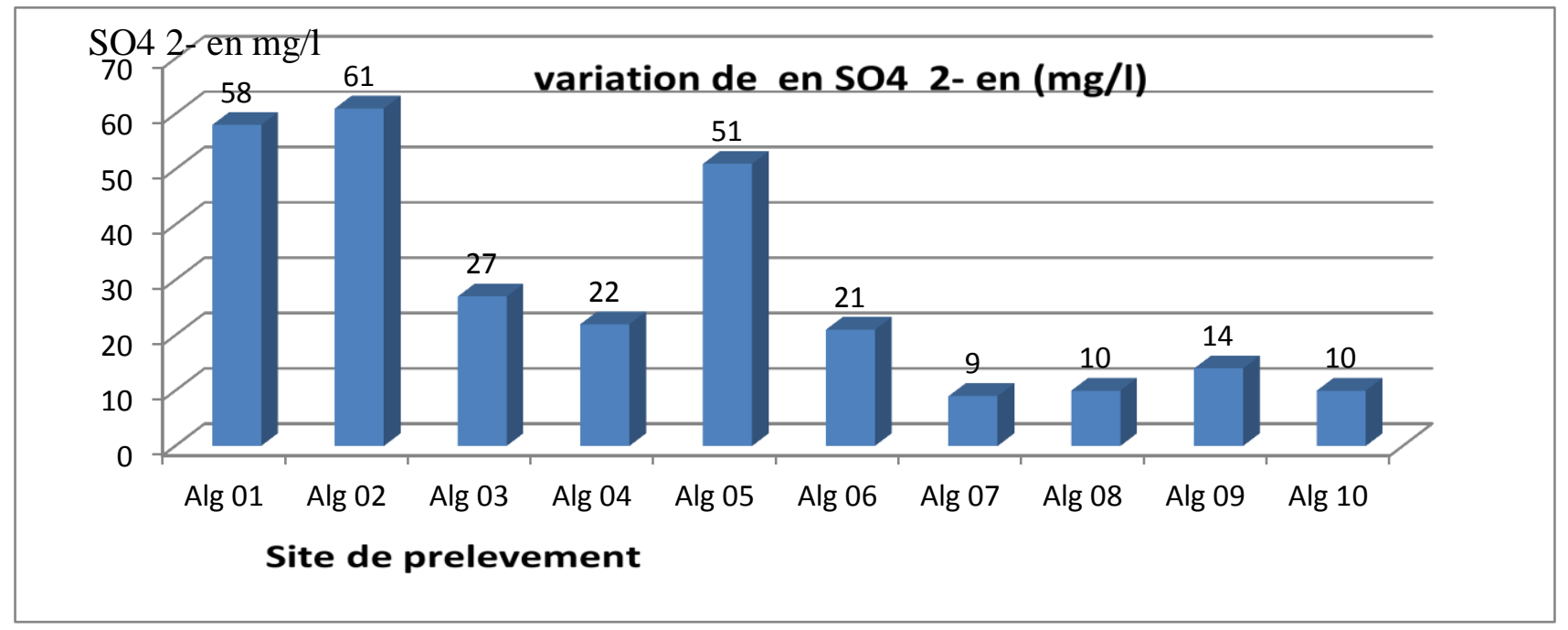

Figure 8 : Variation des sulfates en $\mathrm{mg} / \mathbf{l}$ 
Tableau 5:-Résultats des métaux lourds dans l'eau.

\begin{tabular}{|c|c|c|c|c|}
\hline Paramètres & Unité & Minimum & Maximum & Moyenne \\
\hline Aluminium & $\mathrm{mg} / \mathrm{l}$ & $\mathbf{0 . 0 2}$ & $\mathbf{0 . 1 3}$ & $\mathbf{0 . 0 5}$ \\
\hline Fer & $\mathrm{mg} / \mathrm{l}$ & $\mathbf{0 . 6}$ & $\mathbf{2 . 2}$ & $\mathbf{0 . 6 2}$ \\
\hline Cuivre & $\mathrm{mg} / \mathrm{l}$ & $\mathbf{0 . 1 4}$ & $\mathbf{0 . 0 1 1}$ & $\mathbf{0 . 3 0}$ \\
\hline Manganèse & $\mathrm{mg} / \mathrm{l}$ & $\mathbf{0 . 0 0 5}$ & $\mathbf{1 . 5 2}$ & $\mathbf{0 . 0 0 7}$ \\
\hline Plomb & $\mathrm{mg} / \mathrm{l}$ & $\mathbf{0 . 3 2}$ & $\mathbf{0 . 1 7}$ & $\mathbf{0 . 9 1}$ \\
\hline Cd & $\mathrm{mg} / \mathrm{l}$ & $\mathbf{0 . 0 4}$ & $\mathbf{0 . 0 8}$ \\
\hline
\end{tabular}

En ce qui concerne les métaux lourds tel que ( $\mathrm{Al}, \mathrm{Fe}, \mathrm{Cu}, \mathrm{Mn}, \mathrm{Pb}$ et $\mathrm{Cd}$ ), leurs teneurs sont varies : Les teneurs en Aluminium, Fer, Cuivre, Manganèse, Plomb et Cadinium, des échantillons varient respectivement de $0.02 \mathrm{mg} / \mathrm{L}$ à $0.13 \mathrm{mg} / \mathrm{L}$ et de $0.6 \mathrm{mg} / \mathrm{L}$ à $2.2 \mathrm{mg} / \mathrm{L}$ et $0.14 \mathrm{mg} / \mathrm{L}$ à $0.62 \mathrm{mg} / \mathrm{L}$ et $0.005 \mathrm{mg} / \mathrm{L}$ à $0.011 \mathrm{mg} / \mathrm{L}$ et $0.32 \mathrm{mg} / \mathrm{L}$ à $1.52 \mathrm{mg} / \mathrm{L}$ et $0.04 \mathrm{mg} / \mathrm{L}$ à $0.17 \mathrm{mg} / \mathrm{L}$ (Tableau5).

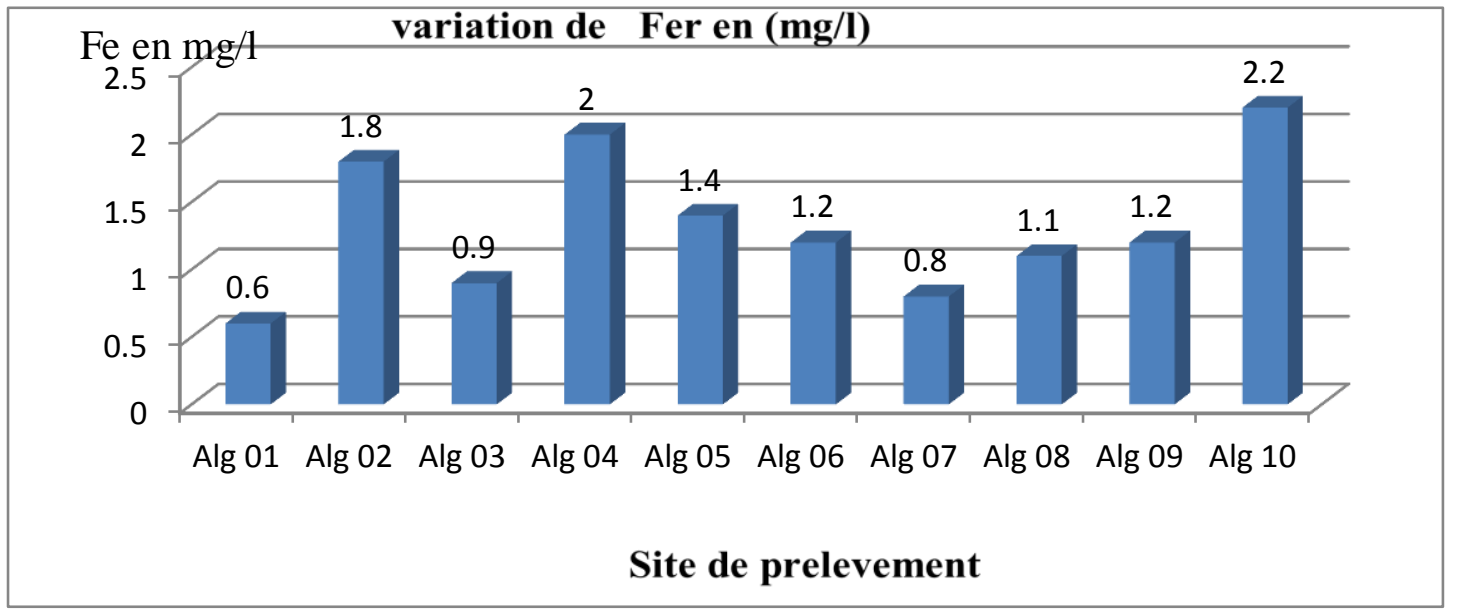

Figure 9 : Variation du fer en $\mathrm{mg} / \mathrm{l}$

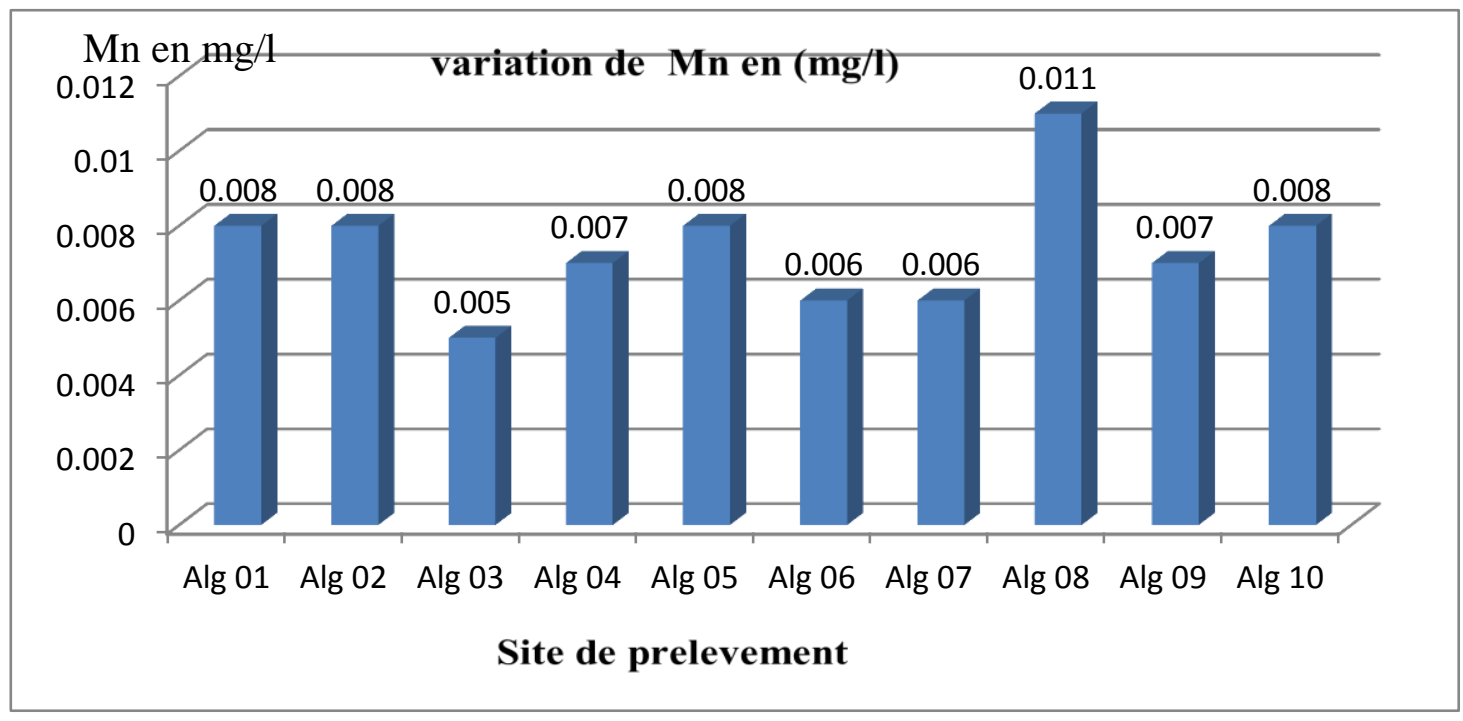

Figure 10 : Variation du Maganées en mg/l 


\section{Discussion:-}

Dans le cadre de notre étude, nous avons effectué au total 10 prélèvements pour l'analyse physico-chimique. Les différents échantillons d'eau brute du lac d'Aleg sont prélevés pour être analysés. Les prélèvements ont été effectués dans des flacons en polyéthylène d'une capacité de 1 litre. A cause des variations qui peuvent affecter les échantillons d'eau pendant le transport, certains paramètres sont déterminés immédiatement sur le site même de prélèvements tels que le $\mathrm{pH}$, la Température, la conductivité électrique, la TDS, et la turbidité.

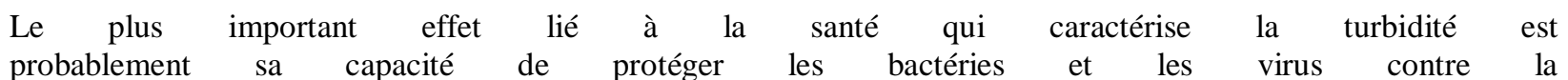
désinfection [3][4].

La turbidité de l'eau brute du lac lors des études avait varié entre 141 NTU et 771 NTU. La présence très élevée des germes indicateurs de la contamination fécale, constituent sans doute une menace pour les habitants qui tirent l'eau nécessaire à la majeure partie de leurs besoins [5], (Tableau2).

Les valeurs repérées sont inférieures à la limite qui est de 5 NTU.

La teneur de l'aluminium a varié au cours de l'étude de $0,02 \mathrm{mg} / \mathrm{L}$ à $0,13 \mathrm{mg} / \mathrm{L}$ (Tableau 5), le taux normal de l'aluminium est fixé par le comité Européenne entre $0,1 \mathrm{mg} / \mathrm{L}$ et $0,2 \mathrm{mg} / \mathrm{L}$.

Au cours de l'étude, il a été observé une variation de la teneur en aluminium lors des prélèvements au niveau des différents sites.

La conductivité électrique traduit le degré de minéralisation globale, elle nous renseigne sur le taux de salinité [6].Les mesures de la conductivité de l'ensemble des échantillons montrent qu'elles sont comprises entre $339 \mu \mathrm{S} / \mathrm{cm}$ et $702 \mu \mathrm{S} / \mathrm{cm}$ (Tableau2).

L'ammonium constitue le produit de la réduction finale des substances organiques azotées et de la matière inorganique dans les eaux. Il provient également de l'excrétion des organismes vivants et de la réduction et la biodégradation des déchets, sans négliger les apports d'origine domestique, industrielle et agricole [7]. Les ions ammonium proviennent de la dégradation des protéines animales (cycle de l'azote), des effluents domestiques (urée) et des ruissellements urbains [2,8]. L'ammonium étant toxique pour l'organisme humain, la présence en quantité importante dégrade la qualité de l'eau. C'est un élément indicateur de la pollution [9]. On remarque que la teneur de l'ammonium a varié au cours de l'étude de $0,00 \mathrm{mg} / \mathrm{L}$ et $0,4 \mathrm{mg} / \mathrm{L}$, le taux normal de l'ammonium est fixé à $0,3 \mathrm{mg} / \mathrm{L}$ selon l'OMS [10].

Au cours de l'étude, il a été observé une variation de la teneur en ammonium lors des prélèvements au niveau des différents sites.

Cette augmentation peut être expliquée par les utilisations du l'eau lac par les animaux.

Les nitrates peuvent être à l'origine de la formation de nitrites et des nitrosamines, responsables de phénomènes potentiellement pathologiques: la méthémoglobinémie et un risque de cancer. La teneur en nitrates a varié au cours de l'étude de 3.8 $\mathrm{mg} / \mathrm{L}$ à $15 \mathrm{mg} / \mathrm{L}$ (Tableau 4).

Les teneurs en nitrates enregistrées au niveau de tous les sites sont très inférieures à $50 \mathrm{mg} / \mathrm{L}$ considérée comme étant la valeur limite pour l'eau potable selon les normes de l'OMS [10].

Les nitrites sont de puissants oxydants qui ont la capacité de transformer l'hémoglobine en méthémoglobine, rendant le sang incapable de transporter l'oxygène jusqu'aux tissus. De tels effets ont été observés chez de nombreuses espèces animales. Les nitrites sont toxiques pour l'organisme humain, sa 
présence en quantité importante dégrade la qualité de l'eau [3]. On remarque que la teneur en nitrites a varié au cours de l'étude de 0,05 à 1,6 mg/L (Tableau 4).

\section{Conclusion:-}

Les résultats de l'analyse des paramètres physico-chimiques de l'eau du lac d'Aleg en Mauritanie présentés dans ce travail, ont montré que le $\mathrm{pH}$ est proche de la neutralité 7.42, la minéralisation est moyenne due à la conductivité avec une moyenne de $452.2 \mu \mathrm{S} / \mathrm{cm}$.

Les valeurs moyennes de la dureté, des ions calcium, magnésium, sodium et le potassium sont respectivement 7.50 ${ }^{\circ} \mathrm{f}, 35.75 \mathrm{mg} / \mathrm{L}, 29.16 \mathrm{mg} / \mathrm{L}, 32 \mathrm{mg} / \mathrm{L}$ et $24.4 \mathrm{mg} / \mathrm{L}$.

En ce qui concerne les composés azotés, les teneurs en nitrates, nitrites et ammonium sont très faibles Les teneurs en Nitrate, Nitrites et Ammonium des échantillons sont respectivement $9.54 \mathrm{mg} / \mathrm{l}$, de $0.475 \mathrm{mg} / \mathrm{L}$ et $0.141 \mathrm{mg} / \mathrm{L}$ (Tableau 4).

les teneurs du sulfates des échantillons sont très faibles sont $28.3 \mathrm{mg} / \mathrm{l}$ (Tableau 4).

En ce qui concerne les métaux lourds Aluminium, Fer, Cuivre, Manganèse, Plomb et Cadinuim, sont très faibles sont respectivement $0.048 \mathrm{mg} / 1,1.32 \mathrm{mg} / 1,0.304 \mathrm{mg} / 1,0.0074 \mathrm{mg} / 1,0.91 \mathrm{mg} / 1$ et $0.078 \mathrm{mg} / \mathrm{l}$ (Tableau 5).

Ainsi, tous les paramètres physicochimiques étudiés, sont compatibles avec les normes des eaux d'alimentation.

\section{References Bibliographiques:-}

1. -AN. TAÏBI, J. GASSANI, AV. ElghADI, A. BALLOUCHE, G. MOGUEDET, ML O. BABA et M O. JIDDOU. Diagnostic de la dynamique des ressources hydriques de surface et des processus «désertification» du lac d'Aleg et de son bassin versant (Brakna, Mauritanie) par télédétection satellite multidate, Télédétection, vol. $5, \mathrm{n}^{\circ}(1-2-3),(2005), \mathrm{p} .123-137$.

2. KM. UDERT, TA. LARESEN, M. BIEBOW and W. GUJER. Urea hydrolysis andprecipitation dynamics in a urine-collecting system. Water Res., 37, (2003), 2571" 2582.

3. B.M.SEMEGA. Interactions physico-chimiques des eaux de la nappe côtière duTrarza (Mauritanie) à Idini et le long du littoral sud. Thèse d'Université, Nice, 1995.

4. SC. JAMES. Metals in municipal landfill leachate and their health effects. Am. J.Public Health, 67, (1977), 429.

5. K. MINT MOHAMED SALEM, AD. N'DIAYE, MOSAO. KANKOU et A. TINE.Evaluation de la qualité de l'Eau de la Rive droite du fleuve Sénégal. Science Lib, 3,110706, (2011), p.12.

6. MOSA. OULD KANKOU. Vulnérabilité des eaux et des sols de la rive droite dufleuve Sénégal en Mauritanie. Thèse de Doctorat, l'université de limoges, option Chimie etMicrobiologie de l'Eau, France, 2004.

7. P. JAGALS, WOK. GRABOW and JC. De VILLIERS. «Evaluation of indicatorsfor assessment of human and animal faecal pollution of surface runoff », Wat. Sci. Tech.,

8. SL. BONTE, M. PONS, O. POTIER and P. ROCKLIN. "Relation betweenConductivity and Ion Content in Urban Wastewater" Journal of Water Science, 21, 4,(2008), 429- 438

9. D. BASSIROU, HO JIDDOU. Colloque international organisé par le Ministère del'Hydraulique et de l'Energie sur Eau, Environnement, Développement Novembre 2002.

10. OMS. Rapport sur la santé dans le monde, résumé d'orientation. <URL:http://www.oms.ch/whr/1998/exum98f.htm, 1996 